Archive for

Organic Chemistry

Arkivoc 2017, part iii, 181-190

\title{
Dearomatization of 3,5-dinitropyridines - an atom-efficient approach to fused 3-nitropyrrolidines
}

Maxim A. Bastrakov, ${ }^{\text {a a }}$ Anna Yu. Kucherova, ${ }^{a}$ Alexey K. Fedorenko, ${ }^{\text {a }}$ Alexey M. Starosotnikov, ${ }^{a}$ Ivan V. Fedyanin, ${ }^{b}$ Igor L. Dalinger, ${ }^{a}$ and Svyatoslav A. Shevelev ${ }^{a}$

${ }^{a}$ N.D. Zelinsky Institute of Organic Chemistry, Russian Academy of Sciences, Leninsky prosp. 47, Moscow 119 991, Russian Federation

${ }^{b}$ A.N. Nesmeyanov Institute of Organoelement Compounds, Russian Academy of Sciences, Vavilova st. 28, Moscow, 119 991, Russian Federation

Email: b max82@mail.ru

Dedicated to Professor Oleg A. Rakitin on the occasion of his 65th anniversary

Received 05-22-2017

Accepted 06-16-2017

Published on line 06-27-2017

\section{Abstract}

An efficient and convenient one-step method for the synthesis of decahydrodipyrrolo[3,4- $\left.b: 3^{\prime}, 4^{\prime}-d\right]$ pyridine derivatives was developed on the basis of 1,3-dipolar cycloaddition of unstabilized $N$-methyl azomethine ylide with 2-substituted 3,5-dinitropyridines. This novel heterocyclic system contains two 3-nitropyrrolidine fragments fused to a partially saturated pyridine ring. Such types of compound, as follows from the literature, can be considered as potential nitric oxide donors.
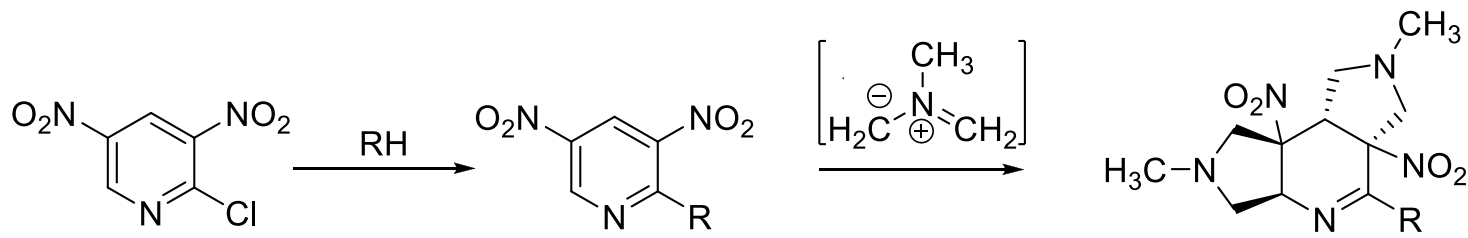

$$
\begin{gathered}
\mathrm{R}=\mathrm{SAr}, \mathrm{SAlk}, \mathrm{OAr} \\
31-68 \%
\end{gathered}
$$

Keywords: Dearomatization, 1,3-dipolar cycloaddition, azomethine ylides, fused pyrrolidines, NO donors, nitrogenoxygen systems, bridgehead nitro groups 


\section{Introduction}

The dearomatization of aromatic compounds is a valuable tool providing a variety of approaches to complex hybrid biologically active molecules and analogues of naturally occurring compounds. ${ }^{1-3}$ In the last few decades, a significant interest in dearomatization of heterocyclic compounds has been observed. ${ }^{2-4}$ This in turn has influenced the development of synthetic organic chemistry: many reactions have been used for carboncarbon and carbon-heteroatom bond formation in the construction of prospective biologically active compounds. ${ }^{4-6}$ Conversion of commercially available aromatics into functionalized alicyclic derivatives is widely used as a strategy for the creation of valuable synthetic products. Among dearomatization methods, 1,3-dipolar cycloaddition is of particular interest. Recently, aromatic substrates (including heterocycles) have been reported to undergo [3+2]-cycloaddition by $\mathrm{C}=\mathrm{C}$ bonds as dipolarophiles with azomethine ylides providing annulation of the pyrrolidine, pyrroline or pyrrole rings. ${ }^{7-10}$

The present work extends our ongoing research ${ }^{11-16}$ on the application of the dearomatization methodology to the synthesis of novel nitrogen-oxygen systems ${ }^{17,18}$ - potential NO donors containing two or more pharmacophoric fragments. Since the 1980s, when the unique role of NO in the regulation of numerous physiological and pathophysiological processes was discovered, this small molecule has been of exceptional interest. ${ }^{19,20}$ Search for new types of NO-donors is one of the actively developing areas of medical chemistry; many papers have been published on the investigation of NO-releasing activity of different classes of compound..$^{21,22}$ For example, recent research shows 3-nitropyrrolidine fused with benzoazoles to be potential NO-donors. ${ }^{23}$ This class of compound is quite limited and therefore insufficiently studied. In addition, the pyrrolidine moiety (including fused pyrrolidines) is considered as a "privileged" structural fragment and a key component of efficient organocatalysts, ${ }^{24}$ enzyme inhibitors, ${ }^{25}$ etc.

Our group has extensive experience in the synthesis of condensed 3-nitropyrrolidines via dearomative 1,3dipolar cycloaddition. Earlier ${ }^{26-29}$ we reported on the first example of [3+2]-cycloaddition of $\mathrm{N}$-methyl azomethine ylide 1 to the benzene ring of a number of $m$-dinitro-benzohetarenes 2 . This resulted in annulation of two pyrrolidine rings to the benzene ring and the formation of polycycles $\mathbf{3}$ (Scheme 1).
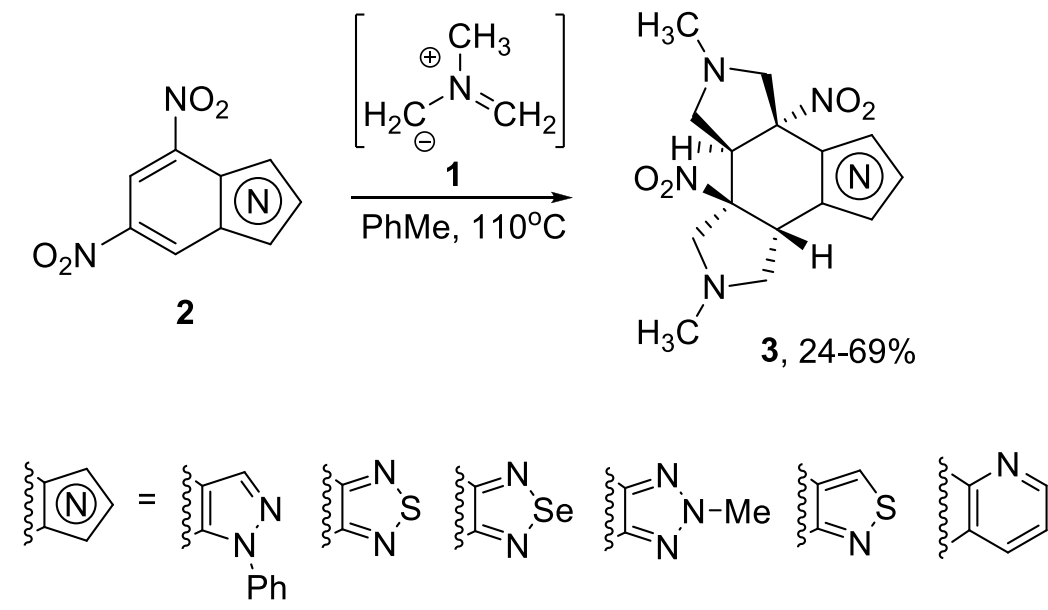

Scheme 1. [3+2]-Cycloaddition of $N$-methyl azomethine ylide to $m$-dinitrohetarenes. 
In addition, it has been reported ${ }^{9}$ that 3,5-dinitropyridine reacts with 3 equivalents of $N$-benzyl azomethine ylide to give the tetracyclic derivative 4 (Scheme 2).
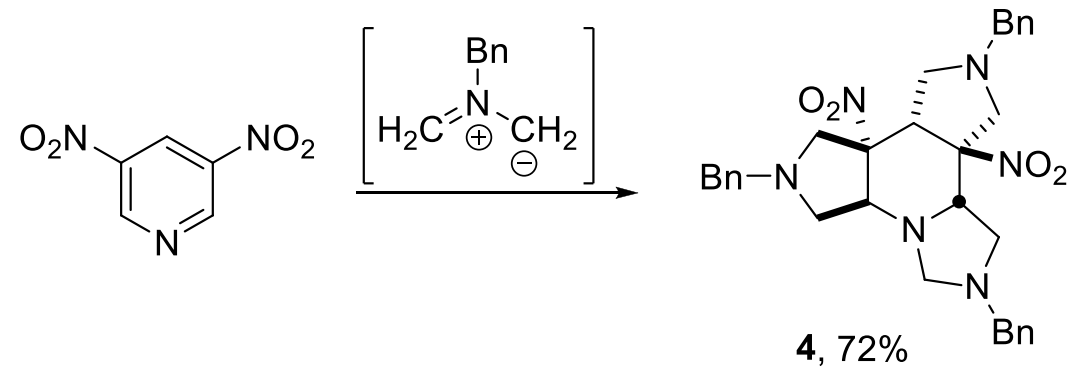

Scheme 2. [3+2]-Cycloaddition of $N$-benzyl azomethine ylide to 3,5-dinitropyridine.

To the best of our knowledge, this reaction constitutes the only example of an uncomplexed pyridine that behaves as a two-electron component in a pericyclic cycloaddition process with azomethine ylides. However, it was found that similar reaction of 3-nitropyridine or its $\mathrm{N}$-oxide with $\mathrm{N}$-benzyl azomethine ylide mainly resulted in the recovery of unconsumed starting material. ${ }^{9}$

\section{Results and Discussion}

Taking into account the above-mentioned results we studied [3+2]-cycloaddition of ylide 1 to 2-substituted 3,5-dinitropyridines. The starting 2-substituted 3,5-dinitropyridines were synthesized from commercially available 2-chloro-3,5-dinitropyridine 5 (Scheme 3). The chlorine atom in this compound can easily be substituted with a variety of nucleophiles. ${ }^{30,31}$<smiles>[R]c1ncc([N+](=O)[O-])cc1[N+](=O)[O-]</smiles>

\begin{tabular}{|c|c|c|c|}
\hline Product & $\mathrm{RH}$ & Reaction conditions & Yield, \% \\
\hline $6 a$ & $\mathrm{PhSH}$ & $\mathrm{Et}_{3} \mathrm{~N}, \mathrm{MeOH}, 20^{\circ} \mathrm{C}, 1 \mathrm{~h}$ & 94 \\
\hline $6 b$ & cyclo- $\mathrm{C}_{6} \mathrm{H}_{11} \mathrm{SH}$ & $\mathrm{Et}_{3} \mathrm{~N}, \mathrm{MeOH}, 20^{\circ} \mathrm{C}, 1 \mathrm{~h}$ & 87 \\
\hline $6 c$ & & $\mathrm{Et}_{3} \mathrm{~N}, \mathrm{MeOH}, 20^{\circ} \mathrm{C}, 1 \mathrm{~h}$ & 83 \\
\hline $6 d$ & $i$-BuSH & $\mathrm{Et}_{3} \mathrm{~N}, \mathrm{MeOH}, 20^{\circ} \mathrm{C}, 1 \mathrm{~h}$ & 81 \\
\hline $6 e$ & $\mathrm{PhOH}$ & $\mathrm{Na}_{2} \mathrm{CO}_{3}, \mathrm{CH}_{3} \mathrm{CN}$, reflux, $2 \mathrm{~h}$ & 93 \\
\hline
\end{tabular}

Scheme 3. Synthesis of 2-substituted 3,5-dinitropyridines. 
Compounds 6a-e were studied in reactions with the unstabilized azomethine ylide 1, which was generated in situ from $\mathrm{N}$-methylglycine and paraformaldehyde (Scheme 1). In all cases the products of double cycloaddition of the dipole to aromatic $\mathrm{C}=\mathrm{C}-\mathrm{NO}_{2}$ fragments were isolated. (Scheme 4)

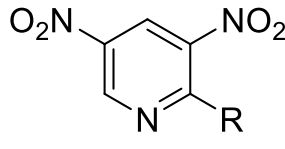

6a-e
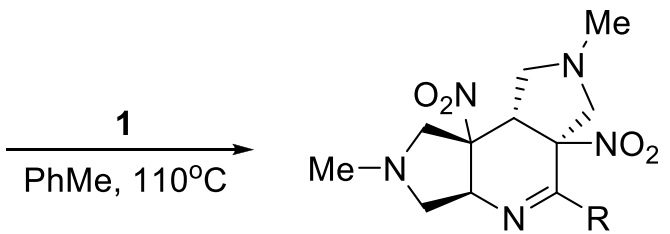

$7 \mathrm{a}, \mathrm{R}=\mathrm{SPh}, 68 \%$

$7 b, \mathrm{R}=\mathrm{S}-\mathrm{C}-\mathrm{C}_{6} \mathrm{H}_{11}, 56 \%$

7c, $\mathrm{R}=\mathrm{SCH}_{2}$ furyl, $50 \%$

$7 \mathrm{~d}, \mathrm{R}=\mathrm{S}-\mathrm{i}-\mathrm{Bu}, 38 \%$

$7 e, R=O P h, 45 \%$

Scheme 4. [3+2]-Cycloaddition of $N$-methyl azomethine ylide to 2-substituted 3,5-dinitropyridines

In contrast to 2-unsubstituted 3,5-dinitropyridine 2 (Scheme 2), the $\mathrm{C}=\mathrm{N}$-bond in compounds 6a-e is unreactive towards azomethine ylide. This result represents the first synthesis of derivatives of novel heterocyclic system - decahydrodipyrrolo[3,4- $\left.b: 3^{\prime}, 4^{\prime}-d\right]$ pyridine. It should be noted that hydrogenated pyrrolo[3,4-c]pyridine core is an important scaffold since compounds of this class possess antibacterial ${ }^{32}$, and anticancer activity ${ }^{33,34}$ as well as cognition activating properties. ${ }^{35}$

The structures of compounds 7a-e were confirmed by NMR-spectroscopy and HRMS data, as well as X-ray diffraction study (for compounds $\mathbf{7 a , b}, \mathbf{e}$ ). It was found that addition of two molecules of the dipole occurs from the opposite sides of the pyridine ring. A similar situation was observed earlier in the case of $m$-dinitrobenzohetarenes, ${ }^{26}$ Scheme 1 . The formation of cycloaddition products 7a-e was found to be diastereoselective. In case of compounds $\mathbf{7 a , b , e}$, the crystal and molecular structure (Figures 1 and 2 ) confirmed the expected cis-addition of the dipole.

In all three compounds studied by X-ray diffraction the annelated five-membered rings and corresponding nitro groups are located on opposite sides of the central heterocyclic fragment. Due to certain steric hindrance, the six-membered rings in $\mathbf{7 a}$ and $\mathbf{7 b}$ have an envelope conformation with atom C5 (Figs. 1 and 2 ) shifted by $0.520(2)$ and $0.473(2) \AA$ from the mean plane of the remaining atoms. Only in $7 e$ is the conformation of the central ring close to a half-chair conformation, characteristic of cyclohexene.

Interestingly, the conformation of the annelated rings adjacent to the nitrogen atom N1 is a perfect envelope with atom C11 out of the plane, and the conformation of the second cycle is intermediate between an envelope and a half-chair. As expected, the nitrogen atoms of the five-membered rings are significantly pyramidalized. Bond lengths and angles in the three compounds are typical to values for related fragments of known substances. The maximal difference is observed for bond N1-C1 that is by $\sim 0.013 \AA$ longer in sulfurcontaining molecules. All interactions between molecules in crystal are of weak van der Waals type. It is worth noting that compounds $\mathbf{7 e}$ and $\mathbf{7 a}$ differ in only one atom (oxygen and sulfur), but they have completely different crystal packing. This fact can be explained by different orientation of the phenyl substituent relative to the central ring: the corresponding torsions N1-C2-O1-C13 and N1-C2-S1-C13 are equal to $-34.26(1)$ and 24.47(1) $)^{\circ}$ and have different signs. 

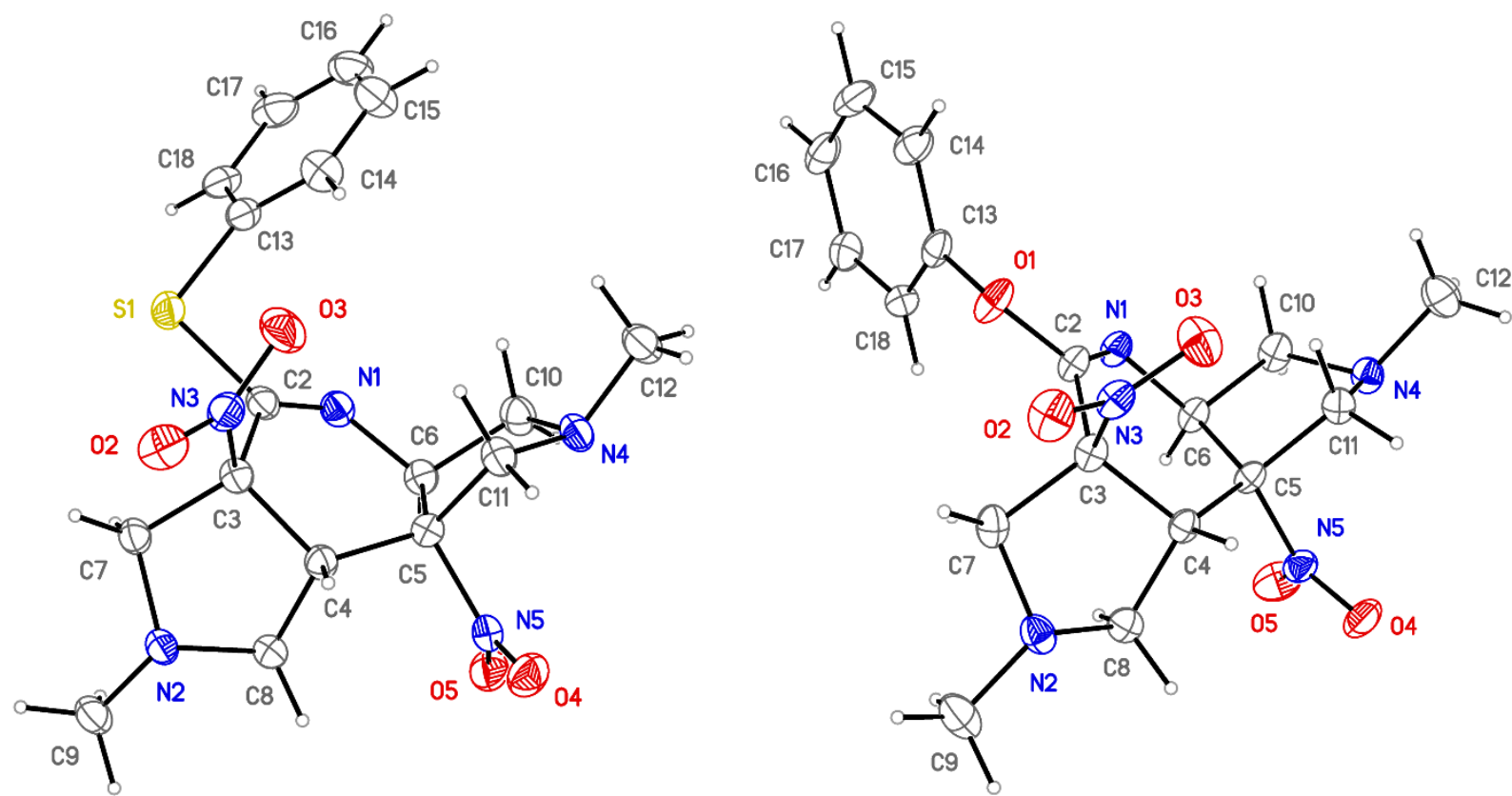

Figure 1. General view of molecules 7a (left) and 7e (right) in the crystal. Anisotropic displacement parameters are drawn with $50 \%$ probability.

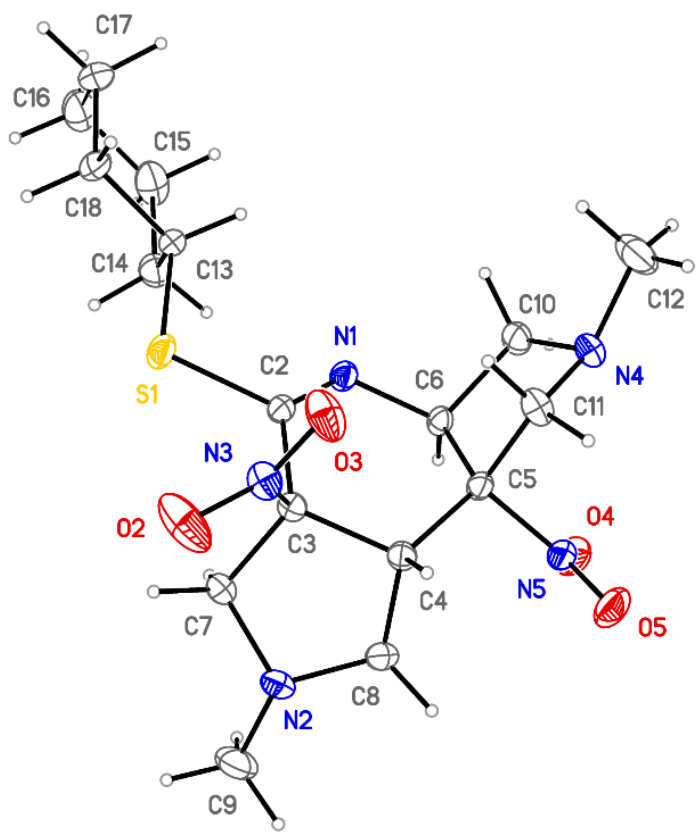

Figure 2. General view of molecule 7b in the crystal. Anisotropic displacement parameters are drawn with $50 \%$ probability. 


\section{Conclusions}

In conclusion, a method for the synthesis of novel polycyclic system - 1,2,3,3a,5a,6,7,8,8a,8b-decahydro-

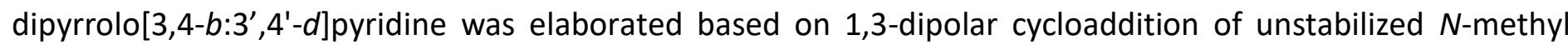
azomethine ylide with 2-R-3,5-dinitropyridines. These compounds represent complex hybrid molecules which can be considered as potential NO-donors having some other types of biological activity due to the presence of pyrrolidine and tetrahydropyridine moieties.

\section{Experimental Section}

General. All chemicals were of commercial grade and used directly without purification. Melting points were measured on a Stuart SMP 20 apparatus. ${ }^{1} \mathrm{H}$ and ${ }^{13} \mathrm{C}$ NMR spectra were recorded on a Bruker AM-300 spectrometer (at 300,13 and 75,13 $\mathrm{MHz}$, respectively) in DMSO- $d_{6}$ or $\mathrm{CDCl}_{3}$ with TMS as internal standard. HRMS spectra were recorded on a Bruker micrOTOF II mass spectrometer using ESI. All reactions were monitored by TLC analysis using ALUGRAM SIL G/UV254 plates, which were visualized by UV light.

X-ray. Data collection for all samples was performed on a Bruker APEX DUO diffractometer equipped with CCD detector (graphite-monochromated MoKa radiation, $\lambda=0.71073 \AA$ or CuKa radiation, $\lambda=1.54178 \AA$ ). Frames were integrated using the Bruker SAINT software package ${ }^{36}$ by a narrow-frame algorithm. A semiempirical absorption correction was applied with the $\mathrm{SADABS}^{37}$ program using the intensity data of the equivalent reflections. The structures were solved with direct methods and refined by the full-matrix least-squares technique against $\mathrm{F} 2 \mathrm{hkl}$ in anisotropic approximation with SHELX ${ }^{38}$ software package. Hydrogen atoms were placed in calculated positions and refined in riding model with Uiso $(\mathrm{Hm})=1.2 \mathrm{Ueq}(\mathrm{Cm})$ for methyl groups and Uiso $(\mathrm{H})=1.2 \mathrm{Ueq}(\mathrm{C})$ for all other hydrogen atoms. Detailed crystallographic information is provided in Supplementary material. Structures were deposited to Cambridge Structural Database, CCDC 15501231550125 contain the supplementary crystallographic data for this paper. These data can be obtained free of charge via http://www.ccdc.cam.ac.uk/data_request/cif , or by e-mailing data_request@ccdc.cam.ac.uk, or by contacting The Cambridge Crystallographic Data Centre, 12 Union Road, Cambridge CB2 1EZ, UK; fax: $+44(0) 1223-336033$.

General procedure for the synthesis of compounds 6a-d. An appropriate thiol $(1 \mathrm{mmol})$ and $\mathrm{Et}_{3} \mathrm{~N}(0.14 \mathrm{~mL}, 1$ $\mathrm{mmol}$ ) were added to a solution of 2-chloro-3,5-dinitropyridine $(0.2 \mathrm{~g}, 1 \mathrm{mmol})$ in methanol $(30 \mathrm{~mL})$. The reaction mixture was stirred at room temperature for $0.5-2 \mathrm{~h}$ until the starting compound was completely consumed (TLC). Then mixture was poured into water and acidified with $\mathrm{HCl}(\mathrm{pH} 4))$. The precipitate that formed was filtered off, washed with water to a neutral reaction and dried in air.

3,5-Dinitro-2-(phenylthio)pyridine (6a). Yellow crystals. Yield 94\%, mp 143-145 ${ }^{\circ} \mathrm{C}$ (lit. ${ }^{30} 123-125{ }^{\circ} \mathrm{C}$ ). ${ }^{1} \mathrm{H}$ NMR $\left(300 \mathrm{MHz}, \mathrm{DMSO}-d_{6}\right): \delta 9.34(\mathrm{~d}, J=1 \mathrm{~Hz}, 1 \mathrm{H}), 9.12(\mathrm{~d}, J=1 \mathrm{~Hz}, 1 \mathrm{H}), 7.62-7.56(\mathrm{~m}, 5 \mathrm{H} \mathrm{Ph}) .{ }^{13} \mathrm{C} \mathrm{NMR}(75 \mathrm{MHz}$, DMSO- $\left.d_{6}\right): \delta 162.7,147.8,140.9,140.2,135.6,130.3,129.7,129.5,127.8$.

2-(Cyclohexylthio)-3,5-dinitropyridine (6b). Yellow crystals. Yield 87\%, mp 130-132 ${ }^{\circ} \mathrm{C} .{ }^{1} \mathrm{H} N M R(300 \mathrm{MHz}$, DMSO- $\left.d_{6}\right): \delta 9.53(\mathrm{~d}, J=2 \mathrm{~Hz}, 1 \mathrm{H}), 9.07(\mathrm{~d}, J=2 \mathrm{~Hz}, 1 \mathrm{H}), 4.09-4.03(\mathrm{~m}, 1 \mathrm{H}), 2.07-1.30(\mathrm{~m}, 10 \mathrm{H}) .{ }^{13} \mathrm{C}$ NMR $(75$ $\mathrm{MHz}$, DMSO- $d_{6}$ ): $\delta 162.6,147.7,140.5,139.9,129.3,43.2,32.0,31.8,25.4,25.0$. Anal. calcd for $\mathrm{C}_{11} \mathrm{H}_{13} \mathrm{~N}_{3} \mathrm{O}_{4} \mathrm{~S}: \mathrm{C}$, 46.64; H, 4.63; N, 14.83; S, 11.32; found C, 46.62; H, 4.61; N, 14.86; S, $11.3 \%$. 
2-[(2-Furylmethyl)thio]-3,5-dinitropyridine (6c). Yellow solid. Yield 83\%, mp 140-141 ${ }^{\circ} \mathrm{C} .{ }^{1} \mathrm{H} \mathrm{NMR}(300 \mathrm{MHz}$, DMSO-d $\left.d_{6}\right): \delta 9.62(s, 1 H), 9.12(s, 1 H), 7.61(s, 1 H), 6.49(s, 1 H), 6.42(s, 1 H), 4.70(s, 2 H) .{ }^{13} \mathrm{C} \mathrm{NMR}(75 \mathrm{MHz}$, DMSO- $d_{6}$ ): $\delta 161.7,149.3,147.7,142.9,140.6,140.5,129.5,110.9,109.2,27.5$. Anal. calcd for $\mathrm{C}_{10} \mathrm{H}_{7} \mathrm{~N}_{3} \mathrm{O}_{5} \mathrm{~S}: \mathrm{C}_{\text {, }}$ 42.71; H, 2.51; N, 14.94; found C, 42.82; H, 2.37; N, 14.72\%.

2-(Isobutylthio)-3,5-dinitropyridine (6d). Brown solid. Yield 81\%, mp 60-61 ${ }^{\circ} \mathrm{C} .{ }^{1} \mathrm{H}$ NMR (300 MHz, DMSO- $\left.d_{6}\right): \delta$ $9.55(\mathrm{~d}, J=2 \mathrm{~Hz}, 1 \mathrm{H}), 9.10(\mathrm{~d}, J=2 \mathrm{~Hz}, 1 \mathrm{H}), 3.26(\mathrm{~d}, J=7 \mathrm{~Hz}, 1 \mathrm{H}), 2.00(\mathrm{~m}, 1 \mathrm{H}), 1.05(\mathrm{~d}, J=7 \mathrm{~Hz}, 6 \mathrm{H}) .{ }^{13} \mathrm{C} \mathrm{NMR}$ (75 MHz, DMSO- $d_{6}$ ): $\delta 163.1,147.6,140.8,140.0,129.3,39.0,27.4,21.8$. Anal. calcd for $\mathrm{C}_{9} \mathrm{H}_{11} \mathrm{~N}_{3} \mathrm{O}_{4} \mathrm{~S}: \mathrm{C}, 42.02$; $H, 4.31 ; N, 16.33$; found C, 42.23; H, 4.19; N, 16.14\%.

3,5-Dinitro-2-phenoxypyridine (6e). Phenol $\left(0.28 \mathrm{~g}, 3 \mathrm{mmol}\right.$ ) and $\mathrm{Na}_{2} \mathrm{CO}_{3}(0.32 \mathrm{~g}, 3 \mathrm{mmol})$ were added to a solution of 2-cloro-3,5-dinitropyridine $(0.61 \mathrm{~g}, 3 \mathrm{mmol})$ in acetonitrile $(50 \mathrm{~mL})$. The reaction mixture was refluxed for $2 \mathrm{~h}$ until the starting compound was completely consumed (TLC), poured into a fivefold excess of water, and acidified with $\mathrm{HCl}$ to $\mathrm{pH}$ 2. The precipitate that formed was filtered off, washed with water to a neutral reaction and dried to give colorless crystals. Yield 93\%, m.p. $163-165{ }^{\circ} \mathrm{C}$ (lit. $\left.{ }^{31} 159{ }^{\circ} \mathrm{C}\right) .{ }^{1} \mathrm{H}$ NMR (300 $\left.\mathrm{MHz}, \mathrm{DMSO}-d_{6}\right): \delta 9.26(\mathrm{~s}, 2 \mathrm{H}), 7.55-7.48(\mathrm{~m}, 2 \mathrm{H}, \mathrm{Ph}), 7.39-7.28(\mathrm{~m}, 3 \mathrm{H}, \mathrm{Ph}) .{ }^{13} \mathrm{C}$ NMR $\left(75 \mathrm{MHz}, \mathrm{DMSO}-d_{6}\right): \delta$ $157.7,152.0,147.8,139.6,131.8,130.1,126.4,121.7$.

General procedure for the synthesis of compounds 7a-e. A mixture of an appropriate 2-substituted 3,5dinitropyridine $(1 \mathrm{mmol})$, paraformaldehyde $(0.18 \mathrm{~g}, 6 \mathrm{mmol})$, and sarcosine $(0.54 \mathrm{~g}, 6 \mathrm{mmol})$ was refluxed in toluene $(30 \mathrm{~mL})$, while adding paraformaldehyde $(0.03 \mathrm{~g}, 1 \mathrm{mmol})$ and sarcosine $(0.089 \mathrm{~g}, 1 \mathrm{mmol})$ every hour until the starting compound was completely consumed (TLC), the mixture was cooled to room temperature. The solvent was evaporated under reduced pressure, and the residue was purified by column chromatography on MN Kieselgel $60\left(0.04-0.063 \mathrm{~mm} / 230-400\right.$ mesh) using $\mathrm{CHCl}_{3}$ as an eluent.

2,7-Dimethyl-5a,8b-dinitro-5-(phenylthio)-1,2,3,3a,5a,6,7,8,8a,8b-decahydrodipyrrolo[3,4- $b: 3^{\prime}, 4^{\prime}$ - $d$ ] pyridine $^{2}$ (7a). Yellow crystals. Yield 68\%, mp 92-94 ${ }^{\circ} \mathrm{C} .{ }^{1} \mathrm{H}$ NMR $\left(300 \mathrm{MHz}, \mathrm{CDCl}_{3}\right): \delta 7.55-7.40(\mathrm{~m}, 5 \mathrm{H}, \mathrm{Ph}), 4.89-4.86(\mathrm{~m}$, $1 \mathrm{H}), 4.23(\mathrm{~d}, J=11 \mathrm{~Hz}, 1 \mathrm{H}), 3.62-3.48(\mathrm{~m}, 3 \mathrm{H}), 3.04-2.91(\mathrm{~m}, 2 \mathrm{H}), 2.65-2.61(\mathrm{~m}, 1 \mathrm{H}), 2.32\left(\mathrm{~s}, 3 \mathrm{H}, \mathrm{CH}_{3}\right), 2.30(\mathrm{~s}$, $\left.3 \mathrm{H}, \mathrm{CH}_{3}\right), 2.15-2.12(\mathrm{~m}, 1 \mathrm{H}) .{ }^{13} \mathrm{C}$ NMR $\left(75 \mathrm{MHz}, \mathrm{CDCl}_{3}\right): \delta 135.2,129.5,129.0,127.0,93.1,92.1,65.7,62.3,61.5$, 61.0, 58.6, 45.3, 41.3, 40.7. HRMS (ESI): $m / z$ calcd for $\mathrm{C}_{17} \mathrm{H}_{21} \mathrm{~N}_{5} \mathrm{O}_{4} \mathrm{~S}+\mathrm{H}^{+}$: 392.1387; found: 392.1392.

5-(Cyclohexylthio)-2,7-dimethyl-5a,8b-dinitro-1,2,3,3a,5a,6,7,8,8a,8b-decahydrodipyrrolo[3,4-b:3',4'-d]pyridine (7b). Brown crystals. Yield 56\%, mp 110-112 ${ }^{\circ} \mathrm{C} .{ }^{1} \mathrm{H}$ NMR $\left(300 \mathrm{MHz}, \mathrm{CDCl}_{3}\right): \delta 4.94-4.96(\mathrm{~m}, 1 \mathrm{H}), 4.15($ $\mathrm{d}, J=12 \mathrm{~Hz}, 1 \mathrm{H}), 3.85-3.49(\mathrm{~m}, 4 \mathrm{H}), 2.95(\mathrm{t}, J=8 \mathrm{~Hz}, 1 \mathrm{H}), 2.45-2.40(\mathrm{~m}, 5 \mathrm{H}), 2.31(\mathrm{~s}, 3 \mathrm{H}), 2.17-1.38(\mathrm{~m}, 10 \mathrm{H}) .{ }^{13} \mathrm{C}$ NMR $\left(75 \mathrm{MHz}_{\mathrm{CDCl}}\right.$ ): $\delta$ 93.8, 92.6, 66.3, 62.4, 60.9, 59.0, 45.2, 43.4, 41.7, 40.9, 32.6, 32.4, 26.0, 25.8, 25.7. HRMS (ESI): $m / z$ calcd for $\mathrm{C}_{17} \mathrm{H}_{27} \mathrm{~N}_{5} \mathrm{O}_{4} \mathrm{~S}+\mathrm{H}^{+}$: 398.1857 ; found: 398.1854 .

5-[(2-Furylmethyl)thio]-2,7-dimethyl-5a,8b-dinitro-1,2,3,3a,5a,6,7,8,8a,8b-decahydrodipyrrolo[3,4- $b$ :3', 4' -

d]pyridine (7c). Brown solid. Yield 50\%, mp 135-136 ${ }^{\circ} \mathrm{C} .{ }^{1} \mathrm{H}$ NMR $\left(300 \mathrm{MHz}, \mathrm{CDCl}_{3}\right): \delta 7.35(\mathrm{~s}, 1 \mathrm{H}), 6.32(\mathrm{~d}, J=3$ $\mathrm{Hz}, 1 \mathrm{H}), 6.22(\mathrm{~d}, J=3 \mathrm{~Hz}, 1 \mathrm{H}), 4.98-5.01(\mathrm{~m}, 1 \mathrm{H}), 4.24(\mathrm{~s}, 2 \mathrm{H}), 4.10(\mathrm{~d}, J=12 \mathrm{~Hz}, 1 \mathrm{H}), 3.77(\mathrm{t}, J=10 \mathrm{~Hz}, 1 \mathrm{H})$, 3.52-3.64 (m, 2H), $3.07(\mathrm{~d}, J=11 \mathrm{~Hz}, 1 \mathrm{H}), 2.95(\mathrm{t}, J=8 \mathrm{~Hz}, 1 \mathrm{H}), 2.60(\mathrm{dd}, J=10 \mathrm{~Hz}, J=3 \mathrm{~Hz}, 1 \mathrm{H}), 2.46(\mathrm{~d}, J=12$ $\mathrm{Hz}, 1 \mathrm{H}), 2.35$ (s, 3H), $2.29(\mathrm{~s}, 3 \mathrm{H}), 2.14(\mathrm{t}, J=10 \mathrm{~Hz}, 1 \mathrm{H}) .{ }^{13} \mathrm{C} \mathrm{NMR}\left(75 \mathrm{MHz}, \mathrm{CDCl}_{3}\right): \delta$ 155.7, 149.7, 142.4, 110.7, 108.4, 93.5, 92.6, 66.2, 62.3, 62.1, 61.0, 58.9, 45.0, 41.3, 40.9, 26.8. HRMS (ESI): $\mathrm{m} / z$ calcd for $\mathrm{C}_{16} \mathrm{H}_{21} \mathrm{~N}_{5} \mathrm{O}_{5} \mathrm{~S}+\mathrm{H}^{+}$: 396.1336; found: 396.1323 .

5-(Isobutylthio)-2,7-dimethyl-5a,8b-dinitro-1,2,3,3a,5a,6,7,8,8a,8b-decahydrodipyrrolo[3,4-b:3',4'-dpyridine (7d). Beige needles. Yield 38\%, mp 98-99 ${ }^{\circ} \mathrm{C} .{ }^{1} \mathrm{H}$ NMR $\left(300 \mathrm{MHz}, \mathrm{CDCl}_{3}\right): \delta 4.95(\mathrm{~m}, 1 \mathrm{H}), 4.18(\mathrm{~d}, \mathrm{~J}=12 \mathrm{~Hz}, 1 \mathrm{H})$, $3.78(\mathrm{~d}, J=10 \mathrm{~Hz}, 1 \mathrm{H}), 3.53-3.58(\mathrm{~m}, 2 \mathrm{H}), 3.11(\mathrm{~d}, J=10 \mathrm{~Hz}, 1 \mathrm{H}) 2.93-3.00(\mathrm{~m}, 2 \mathrm{H}), 2.78-2.85(\mathrm{~m}, 1 \mathrm{H}), 2.59(\mathrm{dd}$, $J=10 \mathrm{~Hz}, J=3 \mathrm{~Hz}, 1 \mathrm{H}), 2.44(\mathrm{~d}, J=12 \mathrm{~Hz}, 1 \mathrm{H}), 2.36(\mathrm{~s}, 3 \mathrm{H}), 2.31(\mathrm{~s}, 3 \mathrm{H}), 2.13(\mathrm{t}, J=10 \mathrm{~Hz}, 1 \mathrm{H}), 1.86(\mathrm{~m}, 1 \mathrm{H})$, 
0.99 (d, $J=6 \mathrm{~Hz}, 6 \mathrm{H}) .{ }^{13} \mathrm{C} \mathrm{NMR}\left(75 \mathrm{MHz} \mathrm{CDCl}_{3}\right): \delta$ 156.9, 93.8, 92.8, 66.2, 62.4, 60.9, 59.0, 45.2, 41.5, 40.9, 38.1, 28.3, 22.0. HRMS (ESI): $\mathrm{m} / z$ calcd for $\mathrm{C}_{15} \mathrm{H}_{25} \mathrm{~N}_{5} \mathrm{O}_{4} \mathrm{~S}+\mathrm{H}^{+}$: 372.1700; found: 372.1688 .

\section{2,7-Dimethyl-5a,8b-dinitro-5-phenoxy-1,2,3,3a,5a,6,7,8,8a,8b-decahydrodipyrrolo[3,4-b:3',4'-d]pyridine}

(7e). Brown crystals. Yield 45\%, mp 112-114 ${ }^{\circ} \mathrm{C} .{ }^{1} \mathrm{H}$ NMR $\left(300 \mathrm{MHz}, \mathrm{CDCl}_{3}\right): \delta 7.39(\mathrm{t}, \mathrm{J}=8 \mathrm{~Hz}, 2 \mathrm{H}), 7.23(\mathrm{t}, \mathrm{J}=$ $7 \mathrm{~Hz}, 1 \mathrm{H}), 7.10(\mathrm{~d}, J=8 \mathrm{~Hz}, 2 \mathrm{H}) 5.02(\mathrm{dd}, 1 \mathrm{H}, J=7,4 \mathrm{~Hz}), 4.13(\mathrm{~d}, J=12 \mathrm{~Hz}, 1 \mathrm{H}), 3.66-3.51(\mathrm{~m}, 3 \mathrm{H}), 3.05-2.88(\mathrm{~m}$,

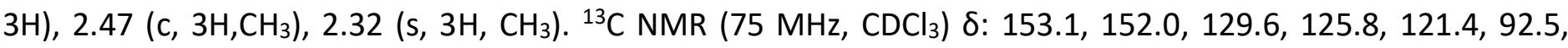
89.3, 64.9, 63.0, 62.4, 59.0, 58.7, 46.8, 41.3, 41.1, 30.9. HRMS (ESI): $\mathrm{m} / z$ calcd for $\mathrm{C}_{17} \mathrm{H}_{21} \mathrm{~N}_{5} \mathrm{O}_{5}+\mathrm{H}^{+}: 376.1615$; found: 376.1607 .

\section{Acknowledgements}

This work was supported by the Russian Science Foundation (grant no. 14-50-00126).

\section{Supplementary Material}

${ }^{1} \mathrm{H},{ }^{13} \mathrm{C}$ NMR and HRMS spectra of compounds 6 and 7, X-ray diffraction data for compounds 7a,b,e.

\section{References}

1. Ramachandran, G.; Sathiyanarayanan, K. I. Curr. Organocatal. 2015, 2, 14. https://doi.org/10.2174/2213337201666141110222735

2. Dudnik, A. S.; Weidner, V. L.; Motta, A.; Delferro, M.; Marks, T. J. Nat. Chem. 2014, 6, 1100. https://doi.org/10.1038/nchem.2087

3. Roche, S. P.; Porco Jr, J. A. Angew. Chem., Int. Ed. 2011, 50, 4068. https://doi.org/10.1002/anie.201006017

4. Smith, P. L.; Chordia, M. D.; Harman, W. D. Tetrahedron 2001, 57, 8203. https://doi.org/10.1016/S0040-4020(01)00805-5

5. Quideau, S.; Pouységu, L. Org. Prep. Proced. Int. 1999, 31, 617. https://doi.org/10.1080/00304949909355348

6. Keane, J. M.; Harman, W. D. Organometallics 2005, 24, 1786. https://doi.org/10.1021/om050029h

7. Shevelev, S. A.; Starosotnikov, A. M. Chem. Heterocycl. Compd. 2013, 49, 92. https://doi.org/10.1007/s10593-013-1233-1

8. Shevelev, S.; Starosotnikov, A. Topics in Heterocyclic Chemistry 2014, 37, 107. https://doi.org/10.1007/7081 2013112

9. Lee, S.; Diab, S.; Queval, P.; Sebban, M.; Chataigner, I.; Piettre, S. R. Chem. Eur. J. 2013, $19,7181$. https://doi.org/10.1002/chem.201201238

10. Roy, S.; Kishbaugh, T. L. S.; Jasinski, J. P.; Gribble, G. Tetrahedron Lett., 2007, 48, 1313. https://doi.org/10.1016/j.tetlet.2006.12.125

11. Bastrakov, M. A.; Starosotnikov, A. M.; Kachala, V. V.; Fedyanin, I. V.; Shevelev, S. A. Asian J. Org. Chem. 
2015, 4, 146.

https://doi.org/10.1002/ajoc.201402207

12. Bastrakov, M. A.; Starosotnikov, A. M.; Fedyanin, I. V.; Kachala, V. V.; Shevelev, S. A. Mendeleev Commun. 2014, 24, 203.

https://doi.org/10.1016/j.mencom.2014.06.004

13. Bastrakov, M. A.; Starosotnikov, A. M.; Kachala, V. V.; Dalinger, I. L; Shevelev, S. A. Chem. Heterocycl. Compd. 2015, 51, 496.

https://doi.org/10.1007/s10593-015-1726-1

14. Steglenko, D. V.; Shevelev, S. A.; Kletskii, M. E.; Burov, O. N.; Lisovin, A. V.; Starosotnikov, A. M.; Morozov, P. G.; Kurbatov, S. V.; Minkin, V. I.; Bastrakov, M. A. Chem. Heterocycl. Compd. 2015, 51, 838. https://doi.org/10.1007/s10593-015-1785-3

15. Starosotnikov, A. M., Bastrakov, M. A., Pavlov, A. A., Fedyanin, I. V., Dalinger, I. L., Shevelev S. A. Mendeleev Commun. 2016, 3, 217.

https://doi.org/10.1016/j.mencom.2016.04.013

16. Bastrakov, M. A.; Starosotnikov, A. M.; Pavlov, A. A.; Dalinger, I. L; Shevelev, S. A. Chem. Heterocycl. Compd. 2016, 52, 690.

https://doi.org/10.1007/s10593-016-1950-3

17. Ananikov, V. P.; Khokhlova, E. A.; Egorov, M. P.; Sakharov, A. M.; Zlotin, S. G.; Kucherov, A. V.; Kustov, L. M.; Gening, M. L.; Nifantiev, N. E. Mendeleev Commun. 2015, 25, 75.

https://doi.org/10.1016/i.mencom.2015.03.001

18. Zlotin, S. G.; Churakov, A. M.; Luk'yanov, O. A.; Makhova, N. N.; Sukhorukov, A. Yu.; Tartakovsky, V. A. Mendeleev Commun. 2015, 25, 399.

https://doi.org/10.1016/i.mencom.2015.11.001

19. Nitric Oxide Donors: For Pharmaceutical and Biological Applications; Wang, P. G., Cai, T. B., Taniguchi, N., Eds.; Wiley-VCH: Weinheim, Germany, 2005.

20. Miller, M. R.; Megson, I.L. Br. J. Pharmacol. 2007, 151, 305.

https://doi.org/10.1038/sj.bjp.0707224

21. Granik, V. G.; Grigoriev, N. B. Nitric oxide (NO). New Route to Drug Design, (in Russian); Vuzovskaya Kniga: Moscow, Russia, 2004.

22. Al-Sa'doni, H.; Ferro, A. Clinic Sci. 2000, 98, 507.

https://doi.org/10.1042/cs0980507

23. Chistyakov, V. A.; Semenyuk, Yu. P.; Morozov, P. G.; Prazdnova, E. V.; Chmykhalo, V. K.; Kharchenko, E. Yu.; Kletskii, M. E.; Borodkin, G. S.; Lisovin, A. V.; Burov, O. N.; Kurbatov, S. V. Russ. Chem. Bull., Int. Ed. 2015, 64, 1369.

24. Sukhorukov, A. Yu.; Sukhanova, A. A.; Zlotin, S. G. Tetrahedron 2016, 72, 6191.

https://doi.org/10.1016/j.tet.2016.07.067

25. Zhmurov, P. A.; Sukhorukov, A. Yu.; Chupakhin, V. I.; Khomutova, Yu. V.; loffe, S. L.; Tartakovsky, V. A. Org. Biomol. Chem. 2013, 11, 8082.

https://doi.org/10.1039/c3ob41646a

26. Bastrakov, M. A.; Starosotnikov, A. M.; Pechenkin, S. Yu.; Kachala, V. V.; Glukhov, I. V.; Shevelev, S. A. J. Heterocycl. Chem. 2010, 47, 893.

https://doi.org/10.1002/ihet.423

27. Konstantinova, L. S.; Bastrakov, M. A.; Starosotnikov, A. M.; Glukhov, I. V.; Lysov, K. A.; Rakitin, O. A.; 
Shevelev, S. A. Mendeleev Commun. 2010, 20, 353.

https://doi.org/10.1016/i.mencom.2010.11.018

28. Bastrakov, M. A.; Starosotnikov, A. M.; Kachala, V. V.; Fedyanin, I. V.; Shevelev, S. A. Asian J. Org. Chem., 2015, 4, 146.

https://doi.org/10.1002/ajoc.201402207

29. Pechenkin, S. Yu.; Starosotnikov, A. M.; Bastrakov, M. A.; Glukhov, I. V.; Shevelev, S. A. Russ. Chem. Bull., Int. Ed. 2012, 61, 74.

30. Biondi, S. Ann. Chim. 1972, 62, 249.

31. Pazek, T. Rocz. Chem. 1960, 34, 165.

32. Altomare, C.; Carotti, A.; Casini, G.; Cellamare, S; Ferappi, M.; Vitali, C. Arzneimittelforschung 1992, 42, 152.

33. Shah, K. R. ; DeWitt Blanton Jr., C. J. Org. Chem. 1982, 47, 502. https://doi.org/10.1021/jo00342a026

34. Geran, R. I.; Greenberg, N. H. ; MacDonald, M. M.; Schumacher, A. M. ; Abbott, B. J. Cancer Chemother. Rep., 1972, 3, 1.

35. Altomare, C.; Carotti, A.; Casini, G.; Cellemare, S.; Ferappi, M.; Gavuzzo, E.; Mazza, F.; Pantaleone, G.; Giorgi, R. J. Med. Chem. 1988, 31, 2153.

https://doi.org/10.1021/im00119a016

36. Bruker, SAINT v7.23A, 2005.

37. G. M. Sheldrick, SADABS v2008/1, Bruker/Siemens Area Detector Absorption Correction Program, 2008.

38. G. M. Sheldrick, Acta Crystallogr., Sect. A: Found. Crystallogr. 2008, 64, 112.

https://doi.org/10.1107/S0108767307043930 